\title{
PALEO
}

Revue d'archéologie préhistorique

$28 \mid 2017$

Varia

\section{Une tête de renne inédite provenant du Magdalénien de Longueroche (Plazac, Dordogne, France)}

An unpublished reindeer head from the Magdalenian of Longueroche

\section{Elena Man-Estier}

\section{OpenEdition}

Journals

Édition électronique

URL : http://journals.openedition.org/paleo/3591

DOI : 10.4000/paleo.3591

ISSN : 2101-0420

Éditeur

SAMRA

\section{Édition imprimée}

Date de publication : 30 décembre 2017

Pagination : 251-255

ISSN : $1145-3370$

\section{Référence électronique}

Elena Man-Estier, «Une tête de renne inédite provenant du Magdalénien de Longueroche (Plazac, Dordogne, France) », PALEO [En ligne], 28 | 2017, mis en ligne le 01 juin 2018, consulté le 07 juillet 2020. URL : http://journals.openedition.org/paleo/3591; DOI : https://doi.org/10.4000/paleo.3591

\section{(c) $(1)(9)$}

PALEO est mis à disposition selon les termes de la licence Creative Commons Attribution - Pas d'Utilisation Commerciale - Pas de Modification 4.0 International 


\title{
Une tête de renne inédite provenant du Magdalénien de Longueroche (Plazac, Dordogne, France)
}

\author{
Elena MAN-ESTIER ${ }^{(a)}$
}

Résumé : La vallée de la Vézère regroupe à la fin du Paléolithique supérieur un ensemble majeur d'occupations à l'envergure et la fonction très différentes. Si le site de La Madeleine est, à juste titre, considéré comme particulièrement important, beaucoup d'autres gisements coexistent aux alentours. En réétudiant progressivement les séries ornées issues des fouilles anciennes, nous nous proposons de contribuer à une discussion renouvelée sur leur place dans ce contexte archéologique particulièrement dense. II en va ainsi de la découverte d'une petite tête de renne gravée sur un fragment osseux issu des fouilles de Denis Peyrony à Longueroche (Plazac). II s'agit de la première représentation figurative de ce site attribuée au Magdalénien supérieur final. À ce titre, elle permet de réinscrire une série modeste et oubliée dans l'important corpus des représentations graphiques de la fin du Tardiglaciaire de la vallée de la Vézère.

Mots-clés : art mobilier, Magdalénien supérieur-final, renne, figuratif.

Abstract: An unpublished reindeer head from the Magdalenian of Longueroche. At the end of Upper Paleolithic, the Vézère valley gathers a major set of occupation whose size and function are different. If La Madeleine may be considered as a real important site, several others co-exist in the neighborhood. By progressive new studies of the decorated collections from former excavations we propose to contribute to a renewed discussion of the status of these occupations in a quite dense archeological background. It is the case for a small reindeer engraved on a bone fragment coming from the Denis Peyrony excavation of Longueroche (Plazac). It appears to be the first figurative image encountered in this site and attributed to Upper Magdalenian. It allows us to re-inscribe this modest and forgotten collection in the important corpus of graphic representations of the end of the late Ice Age in the Vézère valley.

Key-words: portable art, upper Magdalenian, reindeer, figurative.

\section{Historique et contextes archéologiques}

Le site de Longueroche s'ouvre dans un abri-sous-roche, le long d'un petit affluent de la Vézère, le Virmont (fig. 1). II est situé sur la commune de Plazac, à quelques centaines de mètres au nord de la commune du Moustier. L'archéologue suisse-allemand Otto Hauser le loue avant 1914 et y exécute des fouilles qui mettent en évidence des occupations du Magdalénien et de l'Azilien. Les collections qui en résultent ont en partie disparu aujourd'hui (Hauser 1920 ; Zotz 1962-1963). Après l'exil et la déchéance de O. Hauser, Denis Peyrony en reprend la fouille pour le compte de l'État. II effectue deux campagnes de quelques jours en 1932 et 1933 (Peyrony 1932-1933 et Peyrony 1934) dans les témoins non fouillés par $O$. Hauser. II réalise ainsi deux grandes tranchées qui lui permettent de confirmer les occupations précédemment entrevues. Les niveaux identifiés de part et d'autre de l'abri ne sont pas totalement

(a) Direction régionale des Affaires culturelles de Bretagne (Service régional de l'archéologie) et UMR CReAAH 6566 - Université de Rennes 1 - elena.man-estier@culture.gouv.fr 


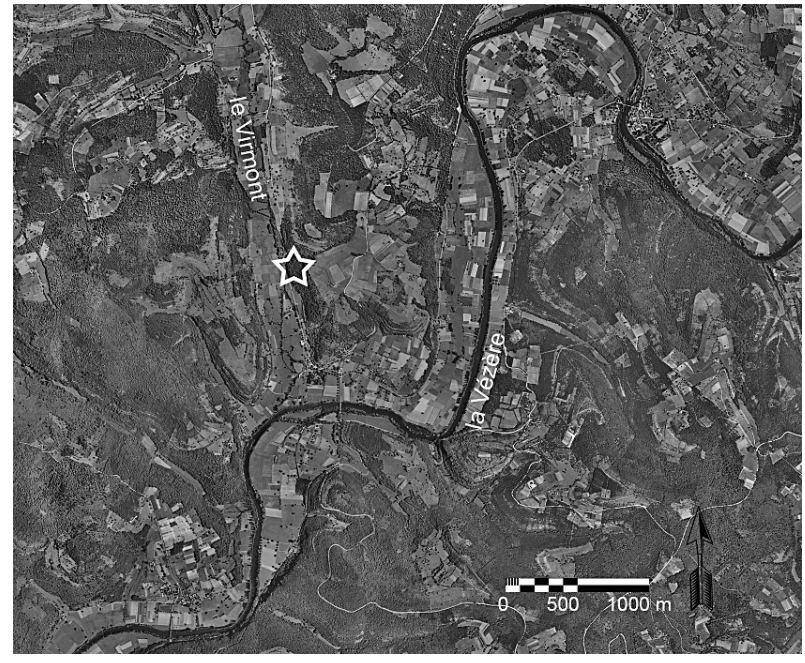

Figure 1 - carte de localisation du site de Longueroche, CAD E. Man-Estier, photographie aérienne des années 1950, (C) IGN, FEDER, Région occitanie, www.geoportail.gouv.fr

Figure 1 - Situation map of Longueroche, DAO E. Man-Estier, Aerial photgraphy of the 50's (O IGN, FEDER, Région occitanie, www.geoportail.gouv.fr

équivalents. Le fouilleur les nomme I (Magdalénien moyen ou Magdalénien III-IV de H. Breuil), II (Magdalénien supérieur ou Magdalénien V) et III (Magdalénien supérieurfinal ou Magdalénien $\mathrm{VI}$ ) dans la tranchée centrale et $\mathrm{A}$ (Magdalénien V), B (Magdalénien VI) et C (Azilien) dans la tranchée nord (fig. 2).

Le premier niveau archéologique identifié est donc un Magdalénien moyen (Magdalénien III-IV de H. Breuil), repéré uniquement au centre de l'abri. II présente une industrie classique de cet horizon culturel avec notamment des sagaies qui évoquent les sagaies Lussac-Angles ("à gouttière sur la face supérieure », (Peyrony 1934 - p. 230 et fig. $4 n^{\circ} 1$ ), des harpons « primitifs » et deux fragments de propulseurs en ronde-bosse. D. Peyrony attribue à ce même niveau un fragment calcaire gravé d'un avant-train de cheval, découvert dans les déblais d'O. Hauser. Cette première occupation de l'abri est suivie par un Magdalénien supérieur (« Magdalénien V »), lui-même immédiatement couronné par un Magdalénien final («Magdalénien VI») (fig. 3). De fines couches stériles marquent de légers hiatus entre les couches. Ces dernières ont des puissances variables, en fonction des coupes et de la zone, de l'ordre de 15 à 25 centimètres. Dans l'ensemble, les séries issues des fouilles $\mathrm{D}$. Peyrony sont typiques des horizons culturels auxquelles elles sont attribuées. C'est par exemple le cas des harpons qui possèdent un, puis deux rangs de barbelures. Les séries de faune indiquent que Cheval et Renne laissent petit à petit place aux petits mammifères

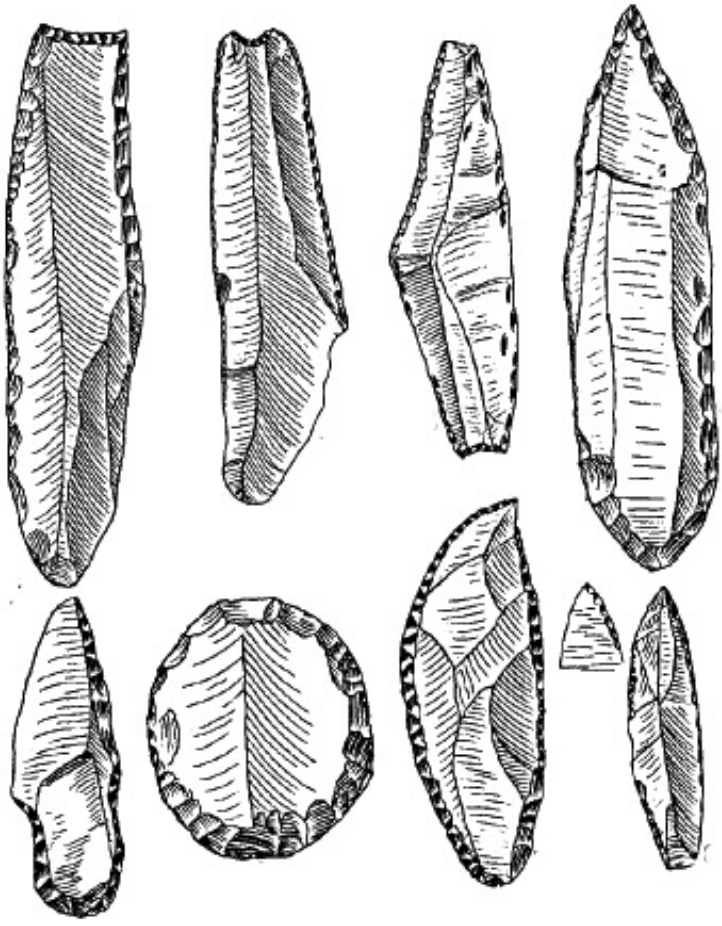

Figure 3 - industrie lithique Magdalénien supérieur-final de Longueroche, Peyrony 1934.

Figure 3 - Lithic industry from Upper Magdalenian of Longueroche, Peyrony 1934.
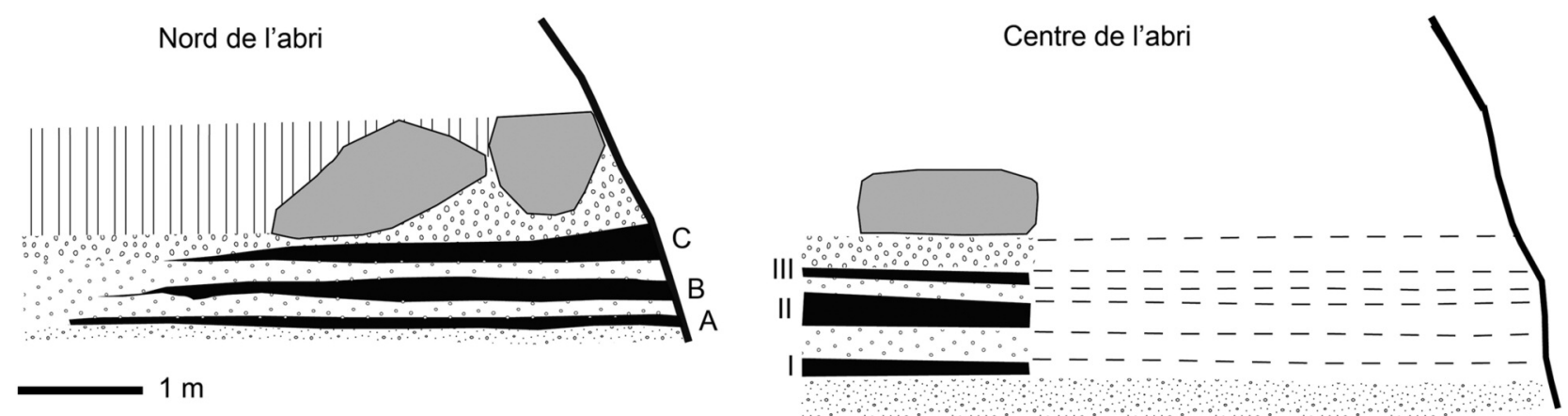

Figure 2 - stratigraphie du gisement de Longueroche, d'après Peyrony 1934, p. 228.

Figure 2 - Stratigraphy of the Longueroche site, after Peyrony 1934. 
typiques de l'Alleröd, au premier rang desquels le Lièvre et le Lapin. Le dernier niveau documenté, dans la partie nord uniquement, est un Azilien « périgourdin ", à bipointes et grattoirs courts, ayant livré à $\mathrm{O}$. Hauser deux harpons plats.

\section{Une nouvelle découverte d'art mobilier}

Il était donc étonnant que ce gisement riche et parfaitement cohérent avec les sites voisins (La Madeleine, Villepin, Laugerie-Basse et Les Marseilles, ou encore la grotte Richard, Raymonden ou La Mairie sont ainsi invoqués par D. Peyrony), n'ait livré qu'une unique représentation figurative, le cheval gravé sur bloc calcaire issu des déblais. À cette exception près, l'ensemble du corpus d'art mobilier était constitué de représentations abstraites, dans lesquelles le fouilleur semble s'être évertué à lire des têtes animales de plus en plus stylisées. Sa démonstration en devient caricaturale quand il évoque, « un dessin du même genre [un animal stylisé] dans lequel l'artiste a poussé la stylisation à tel point qu'il est difficile de l'interpréter » (Peyrony 1934 - p. 241). Avant lui, H. Breuil et R. de SaintPérier ont proposé de lire sur une pièce issue de la fouille Hauser l'image stylisée d'un poisson, sans être plus convaincants (Breuil et Saint-Périer, 1927 - p.88 et fig. 37 $\left.n^{\circ} 3\right)$. II faut dire que ces propositions rejoignent parfaitement les hypothèses formulées par $\mathrm{H}$. Breuil de " dégénérescence » de l'art à l'extrême fin du Magdalénien (Breuil 1905, 1907 ; Man-Estier et al. 2016).
À la faveur d'une révision des séries ornées du Magdalénien supérieur du Périgord', nous avons souhaité réévaluer le potentiel des collections du site. Le corpus comprend désormais 38 pièces ornées, dont seulement 16 ont été publiées (fig. 4). II s'agit presque exclusivement de supports osseux, notamment des pièces d'industrie (pointe de sagaie, baguette demi-ronde) ${ }^{2}$, réparties entre les niveaux du Magdalénien moyen et du Magdalénien supérieur (11 objets dans chaque horizon). Par ailleurs, cinq pièces inédites ne sont pas caractérisées d'un point de vue stratigraphique.

Au sein de la série attribuée au Magdalénien supérieur (sans précision), nous avons eu l'opportunité de découvrir une petite tête de renne gravée sur os. La pièce faisait partie d'un ensemble mélangé de faune et d'industrie osseuse, conservé dans les réserves du Musée national de Préhistoire. La finesse de la gravure explique probablement qu'elle soit passée inaperçue, y compris aux yeux de D. Peyrony qui avait pourtant une grande habitude de lire les gravures sur support mobilier.

La pièce est un métapode de grand herbivore (Boviné ou Cheval) mesurant $12,3 \mathrm{~cm}$ de longueur, $3,6 \mathrm{~cm}$ de largeur et $1,4 \mathrm{~cm}$ d'épaisseur (détermination S. Madelaine, MNP). Elle a été travaillée sur sa face inférieure, qui présente des traces de rainurage. La face supérieure est également marquée par des séries de raclages de la surface et de nombreuses stries de boucherie qui confirment qu'il s'agit

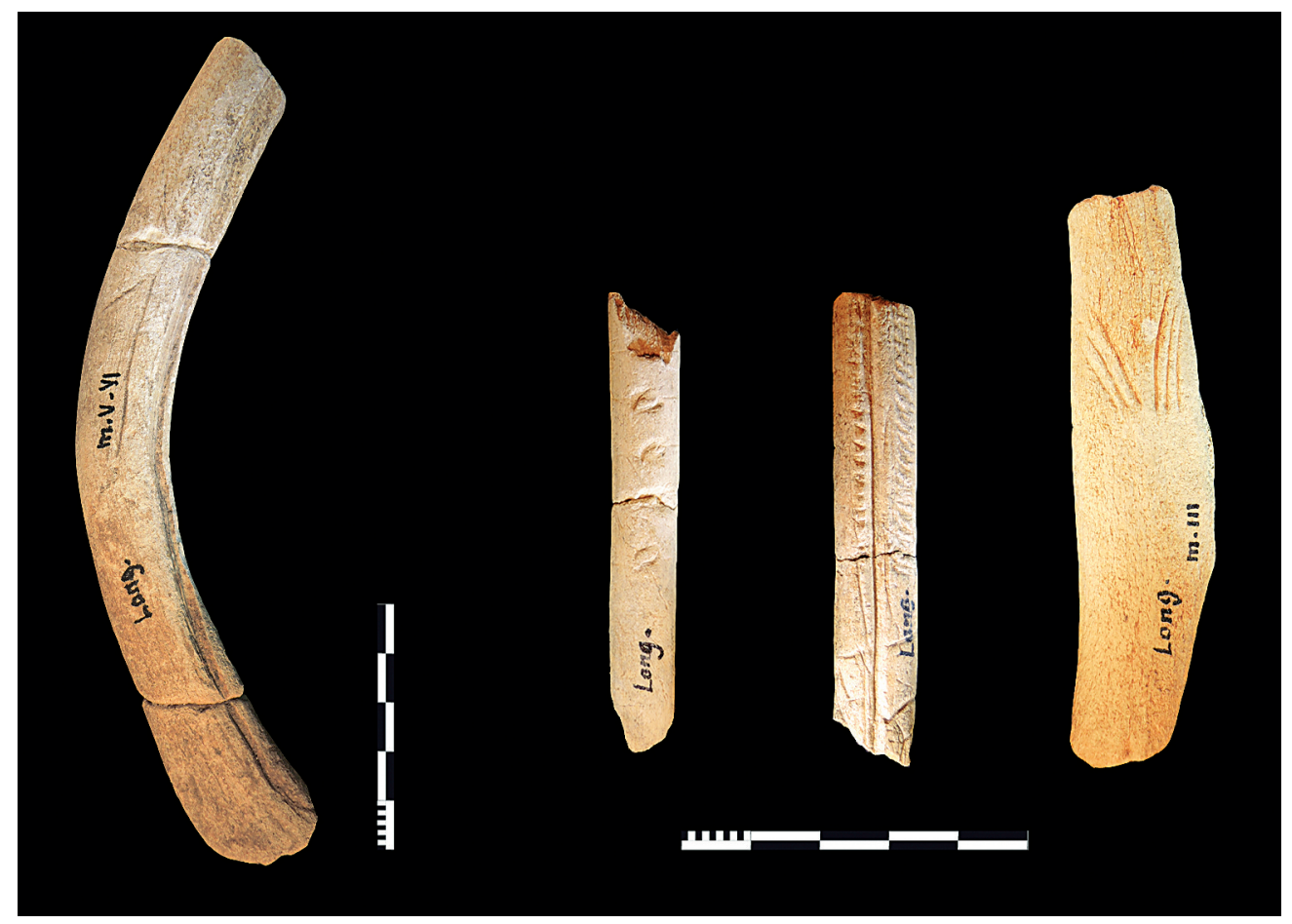

Figure 4 - quelques objets ornés du Magdalénien de Longueroche, clichés E. Man-Estier.

Figure 4 - Some decorated objects from the magdalenian of Longueroche, picture E. Man-Estier.

(1) Menée dans le cadre d'une bourse de recherche octroyée par la fondation Carnot.

(2) Le corpus orné dans sa globalité est en cours d'étude par nous-mêmes et A. Lefebvre. 
avant tout d'un déchet. Cependant, de nombreux stigmates indiquent une utilisation comme compresseur mais il n'est pas possible de les replacer dans une chronologie avec la gravure. Enfin, la pièce est fracturée dans sa partie mésiale, vraisemblablement de manière postérieure à la réalisation de la tête qui est amputée de sa partie arrière (fig. 5).

La tête animale gravée est située dans le coin inférieur droit de la face supérieure et est légèrement débordante sur le flanc. Elle est présentée en profil gauche et la partie arrière est interrompue par la fracture de la pièce. La tête est légèrement relevée et possède un museau rectiligne. Ce dernier est épais, presque camus. II est complété d'un tracé linéaire indiquant le naseau. L'œil, placé en arrière d'une légère inflexion sur la ligne naso-frontale, est traité par un losange encadré de deux courtes parenthèses. Le tracé de la tête est net, d'une grande précision. Sa largeur moyenne est de $0,5 \mathrm{~mm}$. II se distingue sans difficulté des nombreux raclages présents sur le support et tous antérieurs (fig. 6).

La forme du museau et son épaisseur permettent d'identifier sans difficulté un renne. Le traitement de l'œil par des systèmes losangiques concentriques est à rapprocher des nombreuses représentations de cet animal dans le Magdalénien supérieur et final périgourdin, dans l'art mobilier comme dans l'art pariétal. II évoque notamment les gravures fines sur supports stalagmitiques immeubles de la grotte de La Mairie à Teyjat (Paillet et Man-Estier 2016) (fig. 7).

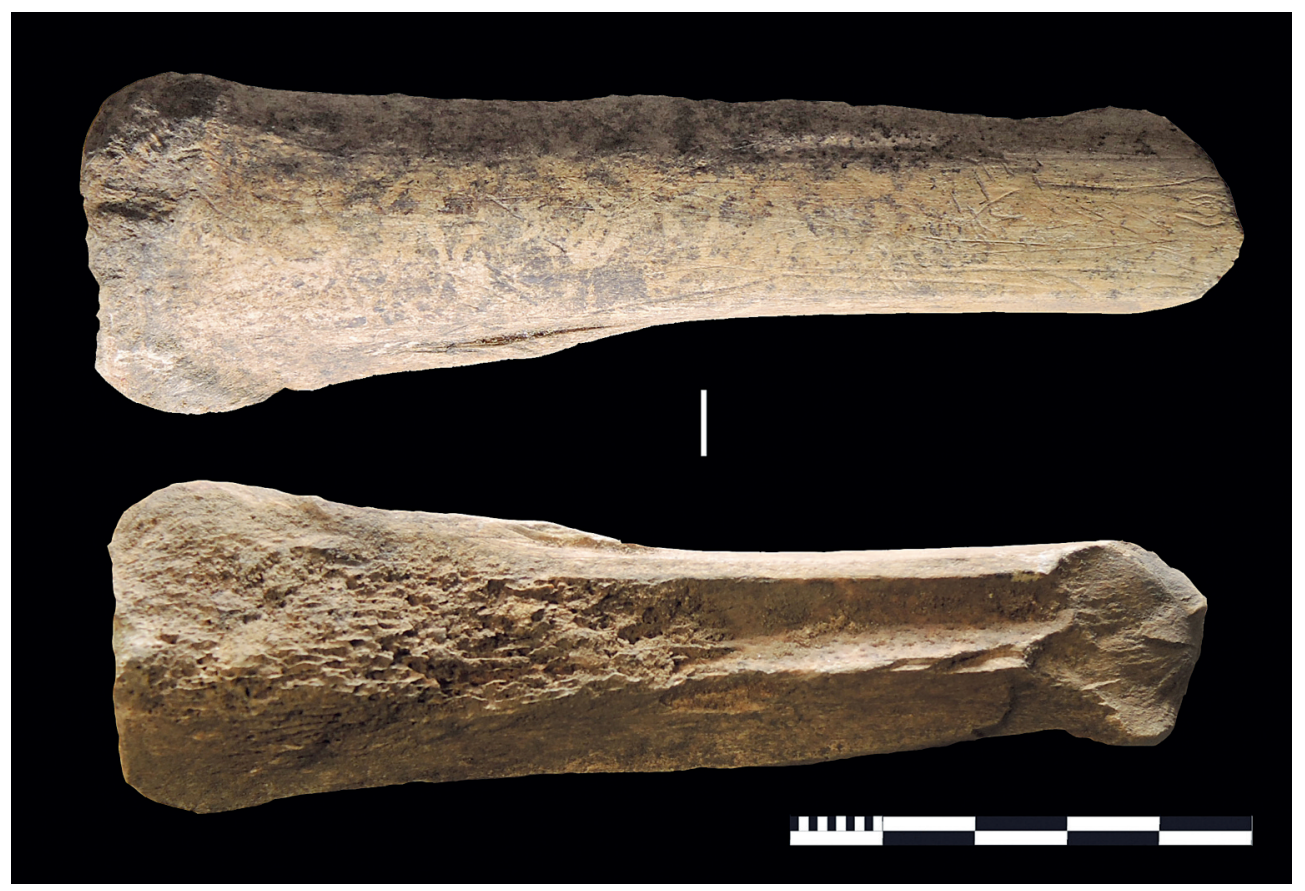

Figure 5 - os gravé, Longueroche, cliché E. Man-Estier.

Figure 5 - Engraved bone, Longueroche, picture E. Man-Estier.
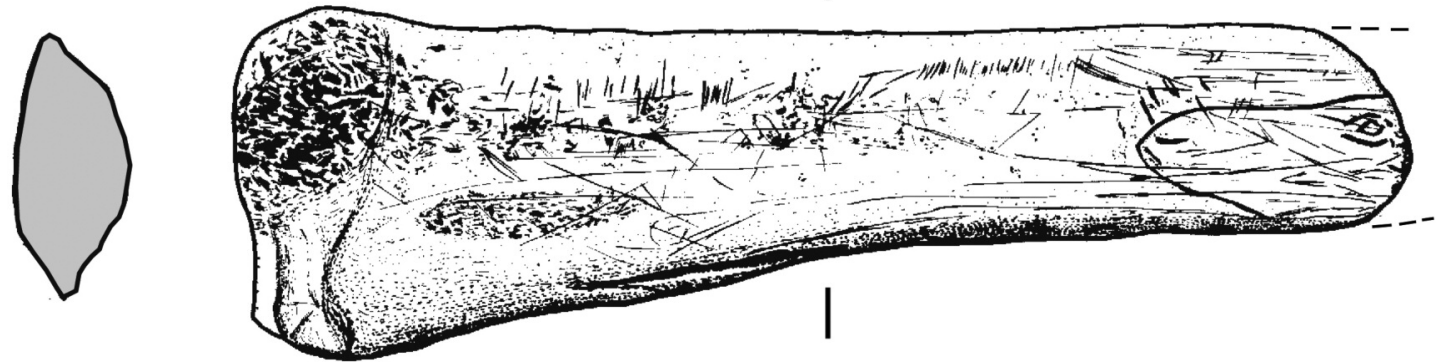

Figure 6 - Os gravé, Longueroche, relevé E. Man-Estier.

Figure 6 - Engraved bone, Longueroche, tracing E. Man-Estier. 


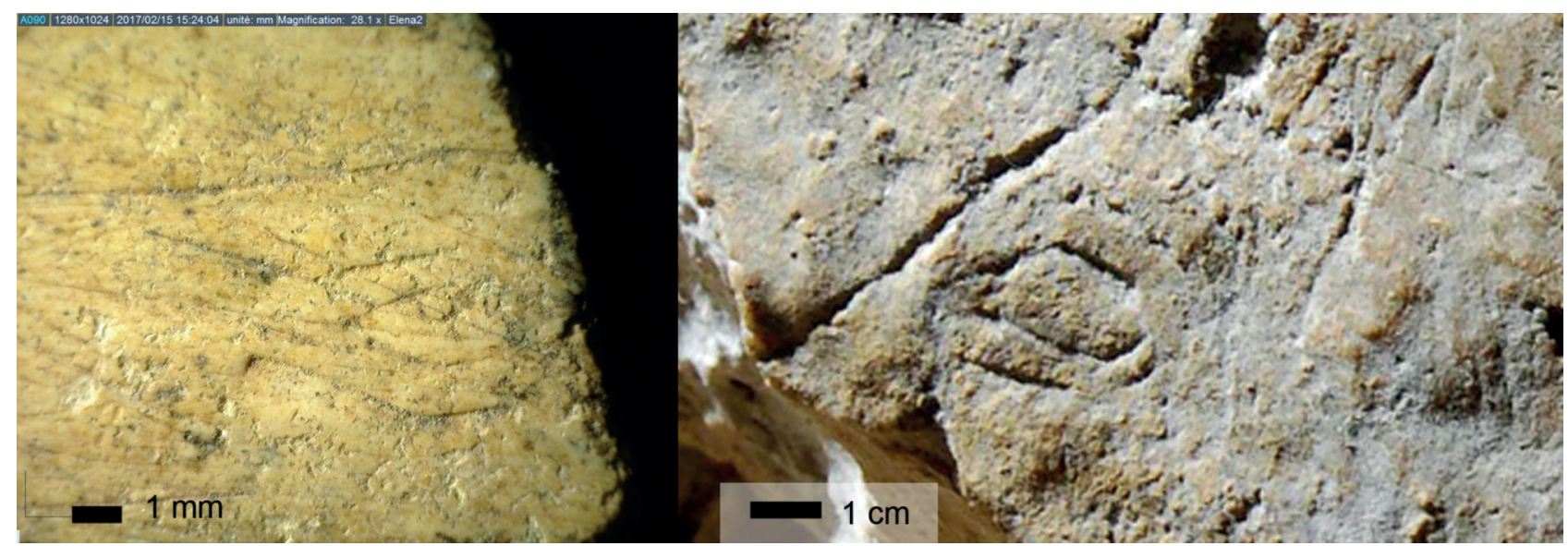

Figure 7 - Détail de l'œil du renne et comparaison avec un renne gravé de la grotte de La Mairie, Teyjat ; cliché P. Paillet, DAO E. Man-Estier.

Figure 7 - Detail of the eye and comparison with engraved reindeer from La Mairie cave ; picture P. Paillet, CAD E. Man-Estier.

Cette petite tête constitue la première représentation figurative du Magdalénien supérieur de Longueroche. Alors que l'on note globalement à cette période une géométrisation et une schématisation des formes, elle rappelle avec d'autres exemples de sites voisins que l'art figuratif ne disparaît pas en un seul temps. Le thème choisi, le Renne, est d'ailleurs emblématique des pulsations climatiques et environnementales que connaissent les derniers magdaléniens. Elles sont également présentes à travers leurs productions symboliques pour lesquelles nous remarquons l'enrichissement du répertoire iconographique par de nouvelles formes toujours plus originales.

Au final, il est important de rappeler combien les séries anciennes sont à même de contribuer à une discussion renouvelée sur les modalités d'occupation à la fin du Tardiglaciaire, et notamment à travers les analyses sur les séries d'art mobilier.

\section{Remerciements}

Ce travail a été financé par la Fondation Carnot dans le cadre d'une bourse de recherches destinée aux Conservateurs du patrimoine. Nous remercions JeanJacques Cleyet-Merle, l'équipe du Musée national de Préhistoire et tout particulièrement C. Boussat, S. Madelaine, P. Jacquement ainsi que M. Langlais, A. Lefebvre et $P$. Paillet pour leurs conseils avisés.

\section{Références bibliographiques}

BREUIL H. 1905 - La dégénérescence des figures d'animaux en motifs ornementaux à l'époque du renne, Compte-rendus de l'Académie des Inscriptions et Belles Lettres, 1905, p. 105-120.

BREUIL H. 1907 - Exemples de figures dégénérées et stylisées à l'époque du renne, Compte-rendus du
XIII Congrès d'Anthropologie et d'Archéologie préhistoriques, Monaco, 1906, p. 394-403.

BREUIL H. et SAINT-PÉRIER R. (de) 1927 - Les poissons, les batraciens et les reptiles dans l'art quaternaire, Masson, archives de l'Institut de Paléontologie humaine, mémoire $\mathrm{n}^{\circ} 2$, Paris.

HAUSER O. 1920 - Ins paradies des urmenschen, Hoffmann Und Campe Verlag, Hambourg et Berlin.

MAN-ESTIER E., PAILLET P., RÉMY D. et VERCOUTÈRE C. 2016 - Supports et représentations dans l'art mobilier magdalénien de Rochereil (Grand-Brassac, Dordogne, France) , In : Cleyet-Merle J.-J., Geneste J.-M., Man-Estier E. (dir.), Actes du colloque « L'art au quotidien Objets ornés du Paléolithique supérieur », Les Eyzies-deTayac, 16-20 juin 2014, Paleo, numéro spécial, 2016, p. 243-260.

PAILLET P. et MAN-ESTIER E. 2016 - « Des animaux et quelques signes à la mairie et à l'abri Mège à teyjat (Dordogne) », In : Groenen M. et M.-C., dir. "Styles, techniques et expression graphique dans l'art sur paroi rocheuse ", British Archeological Records, S2787, actes de la session $A 11 d$ du $17^{e}$ congrès mondial de l'UISPP, Burgos, 2014, pp. 88-120.

PEYRONY D. 1932-1933 - archives inédites, fonds d'archives privées et publiques de D. Peyrony.

PEYRONY D. 1934 - « station préhistorique de Longueroche, Magdalénien et Azilien ", Revue anthropologique, Paris, pp. 226-247.

ZOTZ L. 1962-1963 - «Magdaleoien mit Gigaotolithen von Longueroche an der Vezere », Quartâr 14 Band, Bonn, p. 15-47. 
